\title{
Training and ventilatory assistance in chronic obstructive pulmonary disease
}

\section{To the Editors:}

It was with great interest that I read the paper by VAN'T HUL et al. [1], which was recently published in the European Respiratory Journal, regarding the effectiveness of training with inspiratory support in chronic obstructive pulmonary disease (COPD) patients.

In particular, I longed for an answer to the main question on this topic, i.e. which COPD patients should benefit most from the adjunct of assisted ventilation during training.

Despite brilliant results on the acute effects of assisted ventilation on exercise tolerance and dyspnoea in severe COPD based on a strong pathophysiological background, previous studies on the adjunct of assisted ventilation as an aid to exercise training have yielded conflicting results $[2,3]$.

Even after reading the paper by VAN'T Hul et al. [1], I am not thoroughly convinced that training the patients with all the "paraphernalia" needed for assisted ventilation is worth it. Of course, this does not diminish the value of the study, which is certainly conducted with a rigorous methodological and statistical approach and has added another piece of scientific research on this matter. However, to the extent that every paper has its clinical implications, I wonder if the results of the cited study are somewhat misleading to the readers, causing us to dangerously think that no clinical result can be expected from pulmonary rehabilitation without "mechanical doping".

When analysing the results of the study by VAN'T HuL et al. [1], I am particularly puzzled about the poor results from the rehabilitation programme on exercise tolerance and quality of life obtained in the control group. These results are certainly attributable to the lower intensity of training attained in the control group, particularly when compared to previous studies conducted in COPD patients with comparable severity of disease and trained with traditional methods. Likewise, it is questionable that the between-group difference observed by VAN'T HuL et al. [1] may be attributable to the mere effect of assisted ventilation, since similar results have been shown by EMTNER et al. [4] where the training was supplemented by oxygen, which is a much easier and cheaper "doping".

There is no doubt that exercise training is the cornerstone of pulmonary rehabilitation, even if other complementary treatments are likely to contribute to the final success of a pulmonary rehabilitation programme. Is it possible that the lack of these complementary treatments in the study by VAN'T HuL et al. [1] has contributed to the low rate of success in the control group? Moreover, it is still a matter of debate as to which are the best predictors of pulmonary rehabilitation responders. It is possible that nonresponders to traditional methods of exercise training are those who are most likely to benefit from assisted ventilation; unfortunately, these questions have not been fully addressed in the study by VAN'T Hul et al. [1].

\section{Bianchi}

Respiratory Diseases and Pulmonary Rehabilitation Unit, S. Maugeri Foundation, Institute of Gussago, Gussago, Italy.

\section{REFERENCES}

1 van't Hul A, Gosselink R, Hollander P, Postmus $P$, Kwakkel G. Training with inspiratory pressure support in patients with severe COPD. Eur Respir J 2006; 27: 65-72.

2 Hawkins P, Johnson LC, Nikoletou D, et al. Proportional assist ventilation as an aid to exercise training in severe chronic obstructive pulmonary disease. Thorax 2002; 57: 853-859.

3 Bianchi L, Foglio K, Porta R, Baiardi R, Vitacca M, Ambrosino N. Lack of additional effect of adjunct of assisted ventilation to pulmonary rehabilitation in mild COPD patients. Respir Med 2002; 96: 359-367.

4 Emtner M, Porszasz J, Burns M, Somfay A, Casaburi R. Benefits of supplemental oxygen in exercise training in nonhypoxemic chronic obstructive pulmonary disease patients. Am J Respir Crit Care Med 2003; 168: 1034-1042.

DOI: $10.1183 / 09031936.06 .00005006$

\section{From the authors:}

We would like to thank L. Bianchi for his letter to the editors and AMBROSINO [1] for his editorial regarding our paper on the effects of training with inspiratory pressure support in patients with chronic obstructive pulmonary disease (COPD) [2]. Both reactions focus on the important issue of whether noninvasive ventilatory support (NIVS) during training could now be recommended as an aid in the practical setting of pulmonary rehabilitation.

Is the complex game of assisted ventilation or "mechanical doping" during exercise training in COPD worth the candle? We agree with L. Bianchi that the literature on the effects of training with NIVS in COPD yielded some conflicting results. It must be noted, however, that the study lacking an additional effect of training with NIVS included patients with a relatively mild airway obstruction, well-preserved exercise performance and an absence of inspiratory muscle weakness [3]. In such patients, the ventilatory system is not the primary factor limiting exercise performance. In addition, relatively high levels of ventilation have to be supported, putting a significant demand on the capacity of the ventilator. In the absence of a clear indication for NIVS during exercise or, alternatively, insufficient ventilatory assistance, it is likely that NIVS may work as a deterrent rather than a support to exercise with poor compliance and high drop-out rate as a result. 